\title{
A Gaussian Process Classification and Target Recognition Algorithm for SAR Images
}

\author{
Na Yang and Yongtao Zhang (iD \\ Department of Information and Electronic Engineering, Shangqiu Institute of Technology, Shangqiu 476000, China \\ Correspondence should be addressed to Yongtao Zhang; ytzjw@sqgxy.edu.cn
}

Received 22 November 2021; Revised 20 December 2021; Accepted 4 January 2022; Published 20 January 2022

Academic Editor: Le Sun

Copyright (c) $2022 \mathrm{Na}$ Yang and Yongtao Zhang. This is an open access article distributed under the Creative Commons Attribution License, which permits unrestricted use, distribution, and reproduction in any medium, provided the original work is properly cited.

\begin{abstract}
Synthetic aperture Radar (SAR) uses the relative movement of the Radar and the target to pick up echoes of the detected area and image it. In contrast to optical imaging, SAR imaging systems are not affected by weather and time and can detect targets in harsh conditions. Therefore, the SAR image has important application value in military and civilian purposes. This paper introduces the classification of Gaussian process. Gaussian process classification is a probabilistic classification algorithm based on Bass frame. This is a complete probability expression. Based on Gaussian process and SAR data, Gaussian process classification algorithm for SAR images is studied in this paper. In this paper, we introduce the basic principle of Gaussian process, briefly analyze the basic theory of classification and the characteristics of SAR images, provide the evaluation index system of image classification, and give the SAR classification model of Gaussian process. Taking Laplace approximation as an example, several classification algorithms are introduced directly. Based on the two classifications, we propose an indirect multipurpose classification method and a multifunction classification method for two-pair two-Gaussian processes. The SAR image algorithm based on the two categories is relatively simple and achieves certain results.
\end{abstract}

\section{Introduction}

Synthetic aperture Radar (SAR) is an active modern highresolution microwave imaging Radar [1]. SAR is an active Earth observation system, which can be installed on aircraft, satellites, spacecraft, and other flight platforms to observe the Earth all day and in all weather conditions and has a certain ability to penetrate the surface. SAR improves azimuth and range resolution by using synthetic aperture and pulse compression techniques. It breaks the boundary of Radar camera mode in the past and improves the camera ability of the observation area. Compared with other remote sensing imaging systems, the number of synthetic apertures has increased [2]. The Radar imaging system cannot be affected by light and bad weather and can take necessary ground scenes in one day and in all weather conditions. Because of the above advantages, SAR imaging technology is widely used in resource exploration, land exploration, wartime hostile target inspection, etc. and also has great application to people's livelihood [3]. It has great application potential. When the 5.12 Wenchuan earthquake occurred, it was difficult to obtain rescue information and monitor disasters due to the harsh local climatic conditions. Synthetic aperture Radar can overcome these problems well [4].

With the continuous exploration and development of SAR imaging technology, the demand for SAR image data is getting higher and higher. However, on the other hand, SAR images are quite different from optical images and have the characteristics of correlated noise $[5,6]$. This makes the readability of SAR images much stronger than that of optical images, which makes it difficult for scientific researchers to interpret them manually. Therefore, it is difficult to automatically recognize SAR images at present. Automatic Target Recognition (ATR) technology for SAR images is becoming more and more important and becomes an important research direction [7].

In military warfare, with the development of electronic information countermeasure technology, the outcome of 
electronic warfare has become one of the decisive factors. Especially in the complex electromagnetic environment, it is very important to identify and detect the enemy's intentional camouflage.

Automatic recognition of conventional SAR images requires complex steps such as filter noise reduction, image optimization, and image classification [8]. For automatic recognition of conventional SAR images, complex steps such as image preprocessing, feature extraction, image classification, and recognition are required. The specific process is shown in Figure 1.

There are too many processing steps, and the speed and accuracy cannot meet the actual military needs. Because of the excellent performance of Gaussian process in image processing, SAR image ATR based on Gaussian process has become the focus of research [9]. Gaussian process is a kind of stochastic process in probability theory and mathematical statistics, which makes it easier to classify and segment images. A large number of studies show that Gaussian process technology can be used to effectively process SAR images [10].

\section{SAR Image Classification Algorithm Based on Gaussian Process}

2.1. Research Status of Gaussian Process. Machine learning has been studied for more than a century, and many theories have been formed, but the research on Gaussian process is relatively late. Since 1970s and 1980s, Gaussian process has been applied to geostatistics in the name of Gaussian process regression, such as mineral exploration and remote sensing monitoring [11]. The main problem to be solved is the lowdimensional problem of geoscience statistics, and there is a lack of research on the probability of models and the independence of kernel functions.

Hagan first proposed the use of Gaussian process in the regression model in 1978. Later, Devid J. C. Mackey proposed to establish empirical models by nonlinear parameterization of models, such as radial basis function and layer perceptron. Subsequently, bar Shalom and Fortmann used the Kalman filter to explain the stationary one-dimensional Gaussian process [12].

In 1990s, people made a deeper exploration of kernel function in machine learning. A famous example is support vector machine. At this stage, the application of Gaussian process in machine science has attracted some attention [13]. For example, in 1992, Mackey proposed a framework using Gaussian processes by using approximation methods. However, until 1996, Neal found that its nodes obey Gaussian distribution in the research of Bayesian neural network and Gaussian process began to attract wide attention [14].

In recent years, Gaussian process has been applied to predictive control and soft sensor modeling because of its good model reliability and can meet the requirements of industrial field application. At the same time, it has been further studied in the field of machine learning $[15,16]$.

\subsection{Fundamentals of Gaussian Process}

2.2.1. Machine Learning Theory. Machine learning breeds strong vitality [17]. This is to find out the rules between data by analyzing the characteristics and association of data and to mine all kinds of information needed by human beings at a deeper level. Several common learning methods are briefly introduced as follows:

(1) Description-Based Learning [18]. In a data area, we describe a concrete example, find the main factors associated with the concrete example, and finally perform the process of forming a generalized concept.

(2) Analogical Learning [19]. We compare two data regions, find the similarities between them, and explain the unsolved problems of other data regions from the related theories of one data region [20, 21].

(3) Concentrate on Your Studies. We reason about the existing instances and summarize the specific hypothesis process. From the special to the ordinary, from the simple to the complex, using the local to the whole method, we summarize the learning.

As can be seen from Figure 2, the machine learning system is always on optimized learning mode. By providing back the results from the executive part to the learning part and optimizing the knowledge base all the time, the whole model is optimized. The most important thing in machine learning is to derive learning patterns through training sample data. Therefore, machine learning needs to first determine the learning objects $(X, Y)$ and training samples, learn the sample data with corresponding learning methods, and derive the learning model. Finally, according to the model, a value $Y$ is obtained for any input $X$, which is a vector and contains the hidden information in the data, that is, the necessary information. The mathematical representation of machine learning, which selects the function that best represents the training result from the given $f(x, a)$. The selection is trained only based on the sample data and does not depend on prior knowledge of the sample data that is known in advance. Whether the learning model is suitable to be evaluated by the difference $L(Y, F(X, A))$ between the sample $Y$ value and the value $F(X, A)$ obtained under the machine learning condition is established by

$$
R(a)=\int L(y, f(x, a)) d F(x, y) .
$$

The training sample consists of a probability distribution $F(x, y)=F(x) F(y \mid x)$ composed of $n$ pairs of observed data $\left(x_{1}, y_{1}\right), \ldots,\left(x_{n}, y_{n}\right)$ under independent and identical distribution conditions. In most practical cases, $F(x, y)$ in training is unknown.

The powerful feature extraction ability of CNN depends on the convolution operation of the target image expansion by the convolution kernel. The new value obtained by dot product and sum operation on the image is the eigenvalue of each point pixel [22]. At the level of data structure, both image and convolution kernel are essentially a numerical 


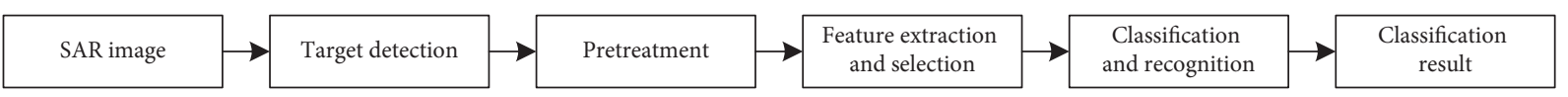

FIgURE 1: SAR automatic recognition flow.

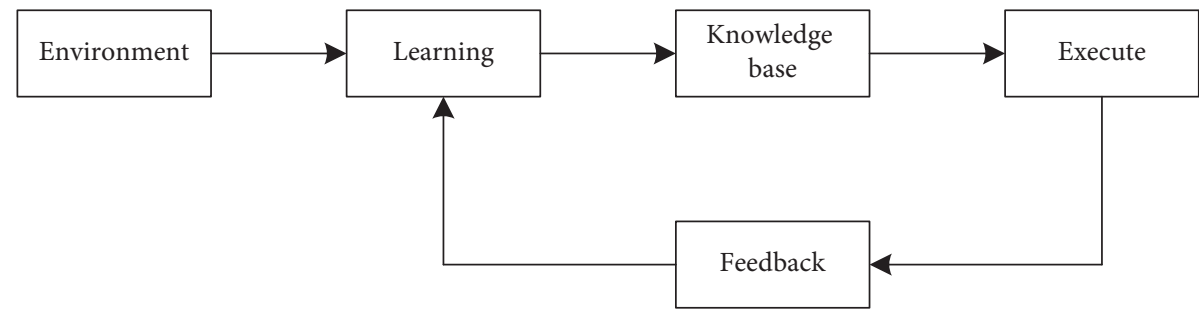

FIGURE 2: Machine learning system model.

matrix, in which the size of image matrix is $W \times W \times D$ and the size of convolution kernel square matrix is $F \times F \times D$. D above represents the depth of the image channel, $W$ represents the side length of the image, and $F$ represents the side length of the convolution kernel. A single channel image with depth 1 and width and height 5 and a convolution kernel with side length 3 are used for convolution operation display. The convolution kernel firstly covers the left vertex area of the feature map and carries out dot product and sum operation on the pixel values in the covered area to obtain the pixel values in the red virtual box in the output feature map, which is the first calculation step of convolution. The green virtual box is the coverage area where the convolution kernel shifts to the right by one unit length and then repeats the dot product and summation operation in the first step to obtain the value of pixels in the green virtual box in the output feature map. Here, the unit length of the convolution kernel shifts to the right is called the moving step size, which is represented by $S$, and this process is the second step of the convolution operation process. When the convolution kernel repeats the above steps and traverses the image matrix from left to right and from top to bottom, it completes a convolution operation process and finally obtains a $3 \times 3$ output feature map.

By substituting the corresponding numerical value, the value of $N$ is 3 , that is, the size of the output characteristic map is $3 \times 3$. It can be seen from the whole convolution operation process that the convolution kernel in each operation process has not changed, and this operation ensures the invariance of the internal parameters of the kernel, that is, weight sharing, which avoids the problem of excessive increase of parameters. In addition, the above convolution operation still brings some problems, such as the reduction of feature image size and insufficient utilization of image edge information. Facing the above problems, we can use filling strategy at the edge of the image to solve them. The edge of the image is filled with pixels with a length of 1 and a value of 0 , and the feature image with a size of $5 \times 5$ can be obtained by recalculating with a convolution kernel with a size of $3 \times 3$, which makes the output image keep the same size as the input image and makes full use of the edge pixels of the original image.
2.2.2. Bayesian Theory. Bayesian theory takes the form of

$$
p(\theta \mid x)=\frac{p(\theta) p(\theta \mid x)}{p(x)}=\frac{p(\theta) p(\theta \mid x)}{\int p(\theta) p(\theta \mid x)} \mathrm{d} \theta .
$$

2.3. Classification Decision Theory. The output of the regression model is converted into class probability by response function, and the following model is adopted:

$$
p\left(C_{1} \mid x\right)=\lambda\left(x^{T} w\right), \lambda(z)=\frac{1}{1+\exp (-z)} .
$$

Up to now, there is no correct answer as to which method to choose. Compared with the direct generation method, the indirect reference method has the advantage that when the modeling of $p(y \mid z)$ is direct, the $x$ dimension is high and the prior density of the class distribution of data is relatively easy to obtain and the practical problem may be more complicated if the direct generation method is adopted, so the indirect reference method is adopted in this paper.

Common optical images are easier to recognize, while SAR images have poor recognition ability because of their features. The synthetic aperture Radar (SAR) imaging system firstly collects the electromagnetic wave reflected from the measured object from the sensor and uses SAR imaging algorithm to reproduce the SAR image in reverse. According to the transmitting and receiving mode of the electromagnetic wave of the sensor, the imaging mode of SAR image can be divided into vertical polarization (V) and horizontal polarization $(\mathrm{H})$. After the two combinations, there are four camera modes: $\mathrm{HH}, \mathrm{HV}, \mathrm{VH}$, and VV. The color optical image has three channels: R, G, and B. SAR images have different channel numbers according to different shooting methods. SAR images are divided into three types according to the number of image channels: unipolarized, dual-polarized, and multipolarized. As the number of channels in a SAR image increases, it contains more information.

The Radar image can be obtained by processing the reflected electromagnetic wave signal of the detected object. However, SAR images have fatal shortcomings. Coherent 
point noise produces interference phenomenon when the target rotates, which leads to anomaly. The gray value of the image shakes violently in a certain range, which is bright and dark, and has a sense of wear. The recognition ability of SAR images is reduced, which makes it difficult to recognize SAR images.

When the surface of the photographing area is uneven, electromagnetic signals reflected from a plurality of different objects are superimposed and returned. Because the surface of each object is uneven, the distance between the SAR imaging system and receiver is random and the signal received by the receiver is related to frequency, but the phase and random change are strong. It is going to return one degree.

Assuming that the imaging area is $K$ scatterers and has an echo reflected on each object, phase $P$, and amplitude $A$, the echo signal received by the receiver after superposition of all scatterer echoes is as follows:

$$
z=A e^{j \varphi}=\sum_{i=1}^{k} A_{i} e^{j \varphi_{i}}
$$

where $A$ and $\varphi$ represent the amplitude and phase of the echo received by the receiver. From the above formula, it is found that when the Radar imaging system moves, the echo path changes and the corresponding phase changes, which affects the whole echo signal. Because of the shortcomings in photography principle, random correlated speckle noise will be produced during photography. In this way, SAR images also produce randomness. On the other hand, SAR images are particularly sensitive to each other's position angle. If the same detected object is shot at different angles, it will cause dramatic changes in SAR images.

There is a very complex correlation between coherent point noise and SAR image information, such as gray value and reflection intensity. $K$ distribution, Weibull distribution, and lognormal distribution, which are often used now, explain the statistical characteristics of SAR images.

2.3.1. K Distribution. For high-resolution SAR images, we will use $K$ distribution to describe its probability density:

$$
p(x)=\frac{2}{b \Gamma(v)}\left(\frac{x}{b}\right)^{v / 2} K_{v-1}\left(2 \sqrt{\frac{x}{b}}\right)(x>0, v>0, b>0),
$$

where $b$ is the scale parameter, $v$ is the shape parameter, and $K_{v}$ is the modified Bassel function of the second kind.

The expectation and variance of the probability density function are as follows:

$$
\begin{aligned}
E(x) & =\frac{b \Gamma(v+(1 / 2)) \Gamma((3 / 2))}{\Gamma(v)}, \\
\operatorname{var}(x) & =b^{2}\left[v-\frac{\Gamma^{2}(v+(1 / 2)) \Gamma^{2}((3 / 2))}{\Gamma^{2}(v)}\right] .
\end{aligned}
$$

2.3.2. Weibull Distribution. Weibull distribution is more suitable for describing SAR images, and its probability density function can be expressed as

$$
p(x)=\frac{c}{b}\left(\frac{x}{b}\right)^{c-1} \exp \left[-\left(\frac{x}{b}\right)^{c}\right] .
$$

Expectations and variances are as follows:

$$
\begin{aligned}
E(x) & =b \Gamma\left(1+c^{-1}\right) \\
\operatorname{var}(x) & =b^{2}\left[\Gamma\left(1+2 c^{-1}\right)-\Gamma^{2}\left(1+c^{-1}\right)\right] .
\end{aligned}
$$

2.3.3. Lognormal Distribution. When SAR imaging, there is a main target to be detected, which is suitable to be described by lognormal distribution. The probability density function is as follows:

$$
p(x)=\frac{1}{x \sigma \sqrt{2 \pi}} \exp \left(\frac{-(\ln (x)-\mu)^{2}}{2 \sigma^{2}}\right),
$$

where $\mu$ denotes scale parameter and $s$ denotes shape parameter. Expectations and variances can be expressed as

$$
\begin{aligned}
E(x) & =e^{\left(\mu+\left(\sigma^{2} / 2\right)\right)}, \\
\operatorname{var}(x) & =e^{2\left(\mu+\sigma^{2}\right)}-e^{\left(2 \mu+\sigma^{2}\right)} .
\end{aligned}
$$

We should use different distribution models to fit the distribution according to the actual situation.

2.4. Classification Accuracy Evaluation System. The classification accuracy of SAR images is mainly based on the measured images on the ground. With the development of SAR remote sensing technology and the complexity of various applications, the accuracy evaluation of SAR image classification becomes more and more important.

2.4.1. Confusion Matrix. The real classification of land surface is expressed by confusion matrix. The values of each column are the same as the true aberration of the surface. The number of categories corresponds to the category image.

2.4.2. Accuracy of Users and Producers. The accuracy of a particular category classification can be determined by calculating the accuracy of a user (UserAcery, UA) and a producer (Producer Accuracy, PA). The specific formula is as follows:

$$
\begin{aligned}
& \mathrm{UA}_{i}=M_{i i} \mid \sum_{j=1}^{T} M_{i j}, \\
& \mathrm{PA}_{i}=M_{i i} \mid \sum_{i=1}^{T} M_{i j} .
\end{aligned}
$$


2.4.3. Kappa Coefficient. The Kappa coefficient can be calculated by the following formula:

$$
K p=\left(n\left(\sum_{i=1}^{T} M_{i i}\right)-\sum_{i=1}^{T}\left(\sum_{j=1}^{T} M_{i j} \sum_{j=1}^{T} M_{j i}\right)\right) \mid\left(n^{2}-\sum_{i=1}^{T}\left(\sum_{j=1}^{T} M_{i j} \sum_{j=1}^{T} M_{j i}\right)\right) .
$$

2.5. SAR Image Classification Algorithm Based on Gaussian Process. Taking the second classification as an example, this section briefly introduces the SAR classification algorithm of Gaussian process. The training data $D=\left(x_{i}, y_{i}\right), i=\{1,2, \ldots, n\}$ is given, the predicted output $y_{*}$ takes into account the target value $x_{*}$ of the sample, and the Gaussian noise usually including zero mean variance $\sigma_{n}^{2}$ is specifically $y=f(x)+\varepsilon$, where $\varepsilon \sim N\left(0, \sigma_{n}^{2}\right)$, that is,

$$
p(y \mid f)=N\left(y \mid f, \sigma_{n}^{2}\right) .
$$

Gaussian process extends multi-Gaussian distribution to an infinite number of random variables. Its kernel function is $k$ :

$$
p(f)=N(0, k) .
$$

Find the edge distribution of $p(y)$ :

$$
p(y)=\int p(y \mid f) p(f) \mathrm{d} f=N(y \mid 0, C) .
$$

When a similar function is configured as a Probit function and the Gaussian result is converted to a probability value, the target variable $y$ follows the Bernoulli distribution:

$$
p(y \mid f)=\sigma(f)^{y}(1-\sigma(f))^{1-y} .
$$

Let $f\left(x_{1}\right), f\left(x_{2}\right) \ldots \ldots f\left(x_{N}\right), f\left(x_{*}\right)$ be denoted as vector $f_{N+1}$. For $f_{N+1}$, the form is

$$
p\left(f_{N+1}\right)=N\left(f_{N+1} \mid 0, C_{N+1}\right),
$$

where

$$
C_{N+1}=\left[\begin{array}{ll}
C_{N} & K \\
K^{T} & C
\end{array}\right]
$$

For the classification problem, the distribution form is

$$
p\left(y_{*}=1 \mid y\right)=\int p\left(y_{*}=1 \mid f_{*}\right) p\left(f_{*} \mid y\right) \mathrm{d} f_{*} .
$$

According to the two classifications, multipurpose classification is carried out. Most of them adopt a pair of multifunctional classifications.

The logarithmic likelihood function of Gaussian process is as follows: $\ln p(y \mid \theta)=-\frac{1}{2} \ln \left|C_{N}\right|-\frac{1}{2} y^{T} C_{N}^{-1} y-\frac{N}{2} \ln (2 \pi)$.

The partial derivative of logarithmic likelihood function for hyperparameters is

$$
\frac{\partial}{\partial \theta_{i}} \ln p(y \mid \theta)=-\frac{1}{2} \operatorname{tr}\left(C_{N}^{-1} \frac{\partial C_{N}}{\partial \theta_{i}}\right)+\frac{1}{2} y^{T} C_{N}^{-1} \frac{\partial C_{N}}{\partial \theta_{i}} C_{N}^{-1} y .
$$

\section{SAR Image Classification Model Based on Multitarget Strategy Gaussian Process}

Most of Gaussian process classification algorithms take two classifications as examples, and SAR images include many target categories. Firstly, this paper introduces the direct multipurpose classification and puts forward the indirect multipurpose classification according to the two classifications.

3.1. Multipurpose Classification Algorithm for Direct Gaussian Processes. Suppose there is a data set $(x, y)$ consisting of $n$ training samples, $x$ is the observation data, and the corresponding category label is $y$, and $f=\left(f_{1}^{1} \ldots f_{n}^{1}, f_{1}^{2} \ldots f_{n}^{2}, \ldots, f_{1}^{C} \ldots f_{n}^{C}\right)^{T}$ is a potential function that divides the whole sample $n$ into vector $C$. In the covariance of $C$, the classification is sometimes denoted by $C$ below. The probability distribution of $F$ is $F \sim \mathrm{N}(0, K)$. It is assumed that the computation between $F$ is independent, $K$ belongs to block diagonal structure, $Y$ is a vector with the same dimension as $F$, and for $I=1, \ldots, N$, its corresponding value is 1 and otherwise, it is 0 .

The output of the Softmax function at training point $i$ is $\pi_{i}^{c}$

$$
p\left(y_{i}^{c} \mid f_{i}\right)=\pi_{i}^{c}=\frac{\exp \left(f_{i}^{c}\right)}{\sum_{c} \exp \left(f_{i}^{c}\right)} .
$$

At this time, a record $c$ with the same length as $f$ is included. The vector of $i$ represents $n$. The multifunctional learning formula is a nonstandard delayed classification label.

$$
\Psi(f)=-\frac{1}{2} f^{T} K^{-1} f+y^{T} f-\sum_{i=1}^{n} \log \left(\sum_{c=1}^{C} \exp \left(f_{i}^{c}\right)\right)-\frac{1}{2} \log |K|-\frac{C_{n}}{2} \log 2 \pi .
$$


Derivation of $f$ on both sides of equation (26) results in

$$
\nabla \Psi=-K^{-1}+y-\pi .
$$

It can be seen from the extreme value formula that when formula (27) is zero, $\widehat{f}=K(y-\widehat{\pi})$ is the maximum value. Derivation is performed again, and it satisfies

$$
-\frac{\partial^{2}}{\partial f_{i}^{c} \partial f_{i}^{c}} \log \sum_{j} \exp \left(f_{i}^{j}\right)=\pi_{i}^{c} \delta_{c c}+\pi_{i}^{c} \pi_{i}^{c}
$$

We have

$$
\nabla \nabla \Psi=-K^{-1}-W
$$

The Newton method is used to search $\psi$, which satisfies the following conditions:

$$
f^{n e w}=\left(K^{-1}+W\right)^{-1}(W f+y-\pi) .
$$

If you need to predict the test point $x_{*}$, you can get a posterior distribution $q\left(f_{*} \mid X, y, x_{*}\right)\left(f\left(x_{*}\right)=f_{*}\right.$ $\left.=\left(f_{*}^{1}, \ldots, f_{*}^{c}\right)^{T}\right)$. In general, use

$$
q\left(f_{*} \mid X, y, x_{*}\right)=\int p\left(f_{*} \mid X, x_{*}, f\right) q\left(f_{*} \mid X, y\right) \mathrm{d} f .
$$

Since $p\left(f_{*} \mid X, x_{*}, f\right)$ and $q\left(f_{*} \mid X, y\right)$ follow Gaussian distribution, $p\left(f_{*} \mid X, x_{*}, f\right)$ follows Gaussian distribution according to the properties of Gaussian distribution. The projected average values for category $C$ are as follows:

$E_{q}\left[f^{c} \mid X, y, x_{*}\right]=k_{c}\left(x_{*}\right)^{T} K_{c}^{-1} f=k_{c}\left(x_{*}\right)^{T}\left(y^{c}-\pi\right)$.

The final equation is obtained from equation (31). By defining the matrix of $C n \times C$,

$$
Q_{*}=\left(\begin{array}{ccc}
k_{1}\left(x_{*}\right) & \cdots & 0 \\
\vdots & \ddots & \vdots \\
0 & \cdots & k_{C}\left(x_{*}\right)
\end{array}\right) .
$$

Equation (32) is transformed into equation (33).

$$
E_{q}\left[f_{*} \mid y\right]=Q_{*}^{T}(y-\pi) .
$$

Or, we can get the following:

$\operatorname{cov}_{q}\left(f_{*} \mid X, y, x_{*}\right)=\operatorname{diag}\left(k\left(x_{*}, x_{*}\right)\right)-Q_{*}^{T}\left(K+W^{-1}\right)^{-1} Q$.

For both classifications, it is necessary to transform the average value of Gaussian distribution. Edge likelihood can be obtained as follows:

$$
\log p(y \mid X, \theta)-\log q(y \mid X, \theta)=-\frac{1}{2} f^{T} K^{-1} f+y^{T} f-\sum_{c=1}^{C} \log \left(\sum_{c=1}^{c} \exp \left(f_{i}^{c}\right)\right)-\frac{1}{2} \log \left|I_{C_{n}}+W^{1 / 2} K W^{1 / 2}\right|
$$

Suppose $W$ is not a diagonal matrix and $K$ is a block diagonal matrix of $C_{n} \times C_{n}$ size. By analyzing the inherent structure of the matrix, it can be modified before using $K^{-1}+W$ :

$$
\left(K^{-1}+W\right)^{-1}=K-K\left(K^{-1}+W\right)^{-1} K
$$

The matrix inversion lemma used in this case is

$$
\left(A^{-1}+B^{-1}\right)^{-1}=A-(A+B) A=B-B(A+B)^{-1} B .
$$

$K^{-1}+W+W$ is inverted. First, the inverse transformation of the $W$ matrix is applied to the matrix of equation (34).

$$
W^{-1}=\left(D-\pi \pi^{T}\right)^{-1}=D^{-1}-R\left(I-R^{-1} K R\right)^{-1} R^{T}=D^{-1},
$$

where $D=\operatorname{diag}(\pi)$; the matrix of $C_{n \times n}$ size composed of the identity matrix of $I_{n}$ is $R=D^{-1} \pi$.

Bring $W^{-1}$ in

$$
\left(K^{-1}+W\right)^{-1}=E-E R\left(\sum_{c} E_{c}\right)^{-1} R^{T} E
$$

3.2. Multipurpose Classification Algorithm for Indirect Gaussian Processes. The one-to-multipurpose classification described in the above section is one of the multipurpose classifications of indirect Gaussian processes. Support vector machine (SVM) can only deal with two classification problems at first, but it cannot deal with many classification problems directly. This section refers to SVM promotion mode and is based on two classification algorithms. The two-to-two Gaussian model of class $C$ classification is shown. This method allows application from two classifications to multiple classifications.

For a given training set $D(X, Y)$ and test data $x_{*}, x=$ $\left[x_{1}, x_{2}, \ldots, x_{n}\right]^{T}$ and $y=\left[y_{1}, y_{2}, \ldots, y_{n}\right]^{T}$. In order to train a classifier between two classes, for $M$ class problems, two classifiers need to be constructed in the training stage. Each classifier collects any two types of data for training. The type of test data $x_{*}$ is determined by the maximum counting method. The method introduced above is fast and has high classification accuracy. The algorithm of this method is as follows:

(1) Initialize the input values for each class

(2) In the training stage, select any two data for training and construct two classifiers

(3) In the training stage, train two classifiers finally between two classes

(4) In the test phase, when the test data $x_{*}$ are classified and discriminated in the training between class $i$ and class $j$, if the output result of these two classifiers is 1 , it belongs to class $i$ of test data $x_{*}$ 


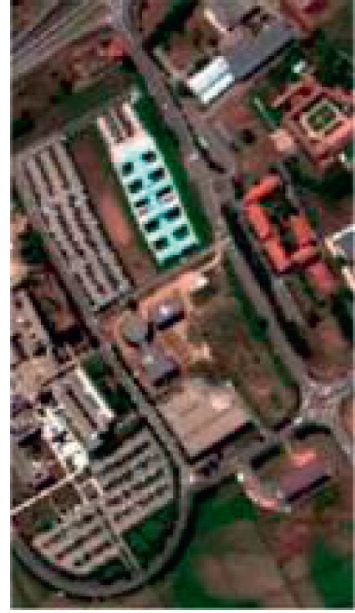

(a)

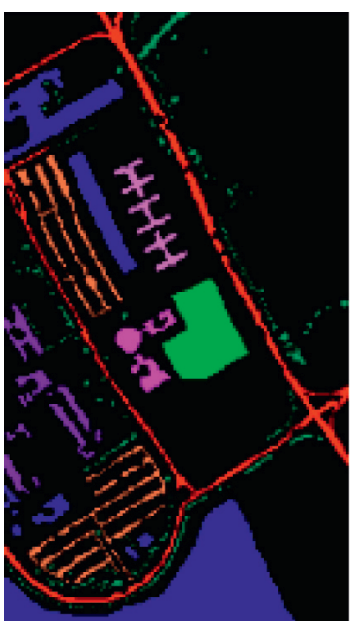

(b)

Figure 3: Pavia University basic information. (a) Original image. (b) Sample distribution.

TABLe 1: Category and feature information of labeled samples at Pavia University and corresponding categories.

\begin{tabular}{lccc}
\hline Category & Ground object information & Number of samples & Proportion of each sample (\%) \\
\hline 1 & Asphalt & 161631 & 15.50 \\
2 & Meadow & 18649 & 43.60 \\
3 & Gravel & 2099 & 4.91 \\
4 & Trees & 3064 & 7.16 \\
5 & Painted metal sheet & 1345 & 3.14 \\
6 & Bare soil & 5029 & 11.76 \\
7 & Asphalt waterproofing & 1330 & 3.11 \\
8 & Brick from necklace & 3682 & 8.61 \\
9 & Shadow & 947 & 2.21 \\
Total & & 42779 & 100 \\
\hline
\end{tabular}
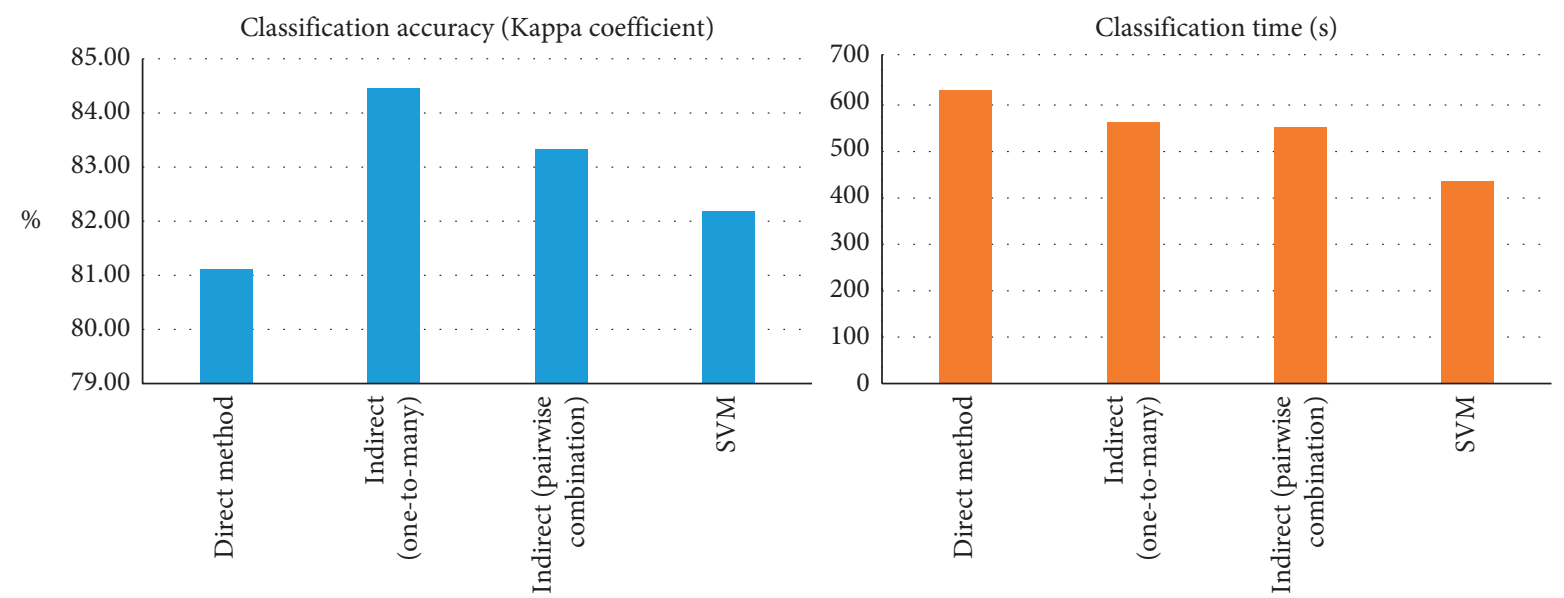

Figure 4: Classification accuracy and time of Pavia University in different ways. In order to analyze and compare more intuitively, compare this figure with classified Figure 5. 


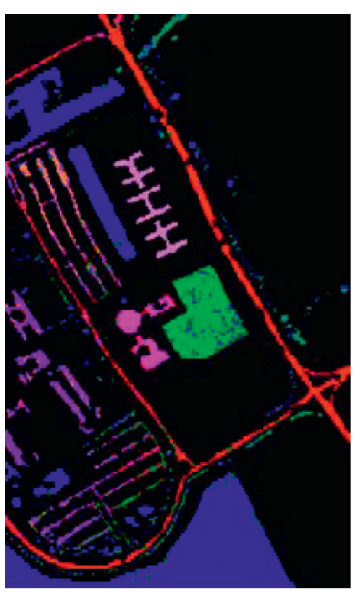

(a)

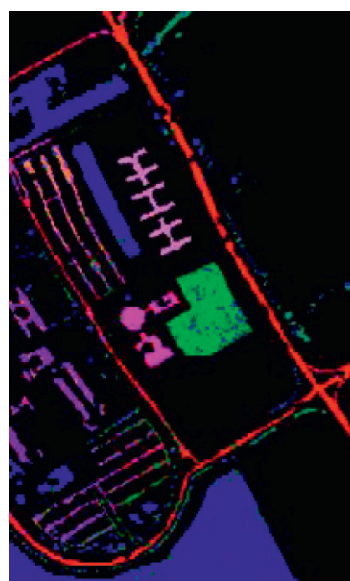

(b)

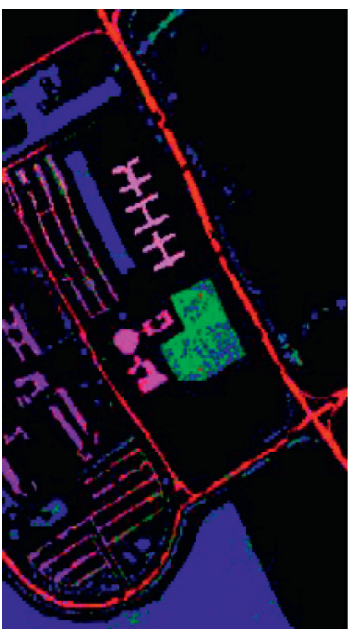

(c)

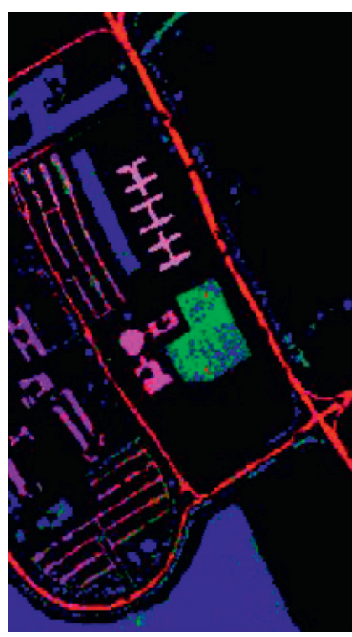

(d)

Figure 5: Pavia University classification renderings. (a) Direct method. (b) Indirect method (one-to-many). (c) Indirect method (two-on-two). (d) SVM.

TABLE 2: Classification results of different methods.

\begin{tabular}{|c|c|c|c|c|c|c|}
\hline Algorithm & 20 & 40 & 80 & 110 & 165 & 220 \\
\hline LNP & - & - & - & 94.11 & 95.97 & 96.05 \\
\hline Triple-GAN & - & - & - & 95.97 & 96.13 & 96.11 \\
\hline Improved-GAN & - & - & - & 95.02 & 97.26 & 98.07 \\
\hline SVM & 76.43 & 87.95 & 92.48 & - & - & - \\
\hline Adaboost & 75.68 & 86.45 & 91.95 & - & - & - \\
\hline GAN & 84.39 & 90.13 & 94.29 & - & - & - \\
\hline MGAN & 85.23 & 90.82 & 94.91 & - & - & - \\
\hline Classification algorithm of indirect Gaussian process & 95.20 & 98.8 & 99.88 & - & - & - \\
\hline
\end{tabular}

During the test phase, the test data $x_{*}$ are calculated. Among the various other numerical values, the largest class of numerical values is the class of $x_{*}$.

\section{Experiment}

The data used in this experiment are from the website of the National University of Bus, Spain. The actual classification samples of the experimental image include 9 kinds of samples, but it can be seen that the data when the samples are discarded are external black stripes.

Figure 3(a) is an original image of Pavia University showing the distribution of the samples shown in Figure 3(b), with irregular land distribution and high degree of crushing. Specifically, the ratio of training samples to prediction samples is about $1: 8$.

Table 1 shows the specific category information and corresponding quantity of samples.

In this experiment, the comparison between indirect multipurpose classification algorithm and support vector machine verifies the direct multipurpose classification algorithm. The experimental results are shown in Figure 4. The approximation algorithms used in experiments are all Laplace approximation methods.

As can be seen from Figures 4 and 5, even if the direct or indirect Gaussian process is applied to SAR classification, the

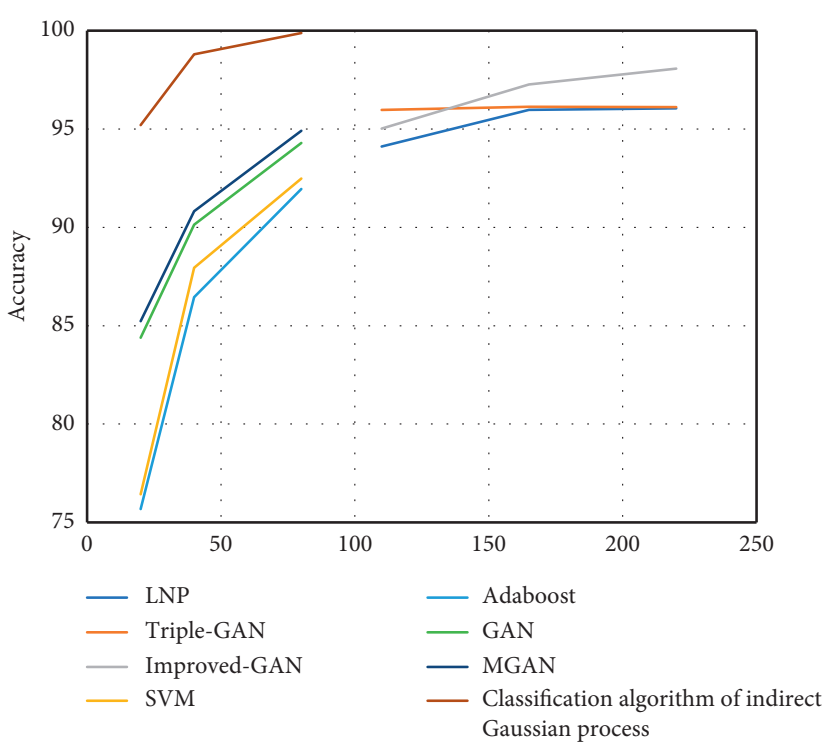

Figure 6: Classification results of various methods.

classification accuracy is better than SVM. It is better to classify more kinds of SAR than to classify SAR directly according to the indirect strategy of two classifications. The classification accuracy of SAR images is preferably one-to-many. 
This paper compares the classification methods for evaluating performance in other MSTAR datasets including LNP, imported-GAN, MGAN, and triple-GAN. LNP assumes that each data point can obtain information from adjacent data and reconstruct it linearly. Triple-GAN uses three network modules, discriminator, generator, and classifier, to achieve the best results of the deep countermeasure model. Indirect Gaussian process classification algorithm achieves the best results in classification and can use the latest training model. MGAN improves images generated using dynamically tuned multirecognizer architecture and improves classification accuracy. The results of typical machine learning methods, for example, SVM and Adaboost, are compared. In the comparative experiment, the same number of labeled samples were used to randomly select samples, and the experimental results were compared with the quantitative results of the paper. The method we use is indirect Gaussian process classification algorithm. The comparison results are shown in Table 2. Figure 6 shows the classification accuracy of methods with different amounts of tag training data.

\section{Conclusion}

This paper describes the characteristics of SAR images and the difficulty of processing and extracts the advantages of Gaussian process classification in SAR images. Finally, the evaluation index of image classification is given, and a certain standard of Gaussian SAR classification algorithm is given to evaluate the classified image and its basic program is introduced.

Based on the analysis and introduction of machine learning theory and Bessel principle, this paper expounds the basic principle of Gaussian process. This paper introduces the basic theory of classification, then leads to the fact that classification usually needs to be solved, and discusses it in detail. This paper describes the characteristics of SAR image and the difficulty of processing and extracts the advantages of Gaussian process classification in SAR image processing. Finally, we give the evaluation index of image classification, give a certain standard of Gaussian process SAR classification algorithm to evaluate the classified image, and introduce its basic program.

\section{Data Availability}

The experimental data used to support the findings of this study are available from the corresponding author upon request.

\section{Conflicts of Interest}

The authors declare that they have no conflicts of interest regarding this work.

\section{Acknowledgments}

This work was sponsored in part by Henan Province Higher Education Training Program for Young Core Teachers, under Grant no. 2021GGJS192.

\section{References}

[1] M. Stasolla, J. J. Mallorquí, G. Margarit, C. Santamaria, and N. Walker, "A comparative study of operational vessel detectors for maritime surveillance using satellite-borne synthetic aperture radar," IEEE Journal of Selected Topics in Applied Earth Observations and Remote Sensing, vol. 9, no. 6, pp. 2687-2701, 2019.

[2] G. Shu, N. Wang, W. Wang et al., "A novel vortex synthetic aperture radar imaging system: decreasing the pulse repetition frequency without increasing the antenna aperture," IEEE Transactions on Geoscience and Remote Sensing, vol. 60, pp. 1-14, 2022.

[3] Y. Xiang, "Exploration of the application of artificial intelligence technology in mechatronics technology based on," Journal of Physics: Conference Series, vol. 1915, no. 2, Article ID 22059, 2021.

[4] S. K. Ahmad, F. Hossain, H. Eldardiry, and T. M. Pavelsky, "A fusion approach for water area classification using visible, near infrared and synthetic aperture radar for south asian conditions," IEEE Transactions on Geoscience and Remote Sensing, vol. 58, no. 4, pp. 2471-2480, 2020.

[5] E. Shah, P. Jayaprasad, and M. E. James, "Image fusion of SAR and optical images for identifying antarctic ice features," Journal of the Indian Society of Remote Sensing, vol. 47, no. 12, pp. 2113-2127, 2019.

[6] L. Wan, Y. Xiang, and H. You, "An object-based hierarchical compound classification method for change detection in heterogeneous optical and SAR images," IEEE Transactions on Geoscience and Remote Sensing, vol. 57, no. 12, pp. 9941-9959, 2019.

[7] L. Wang, X. Bai, C. Gong, and F. Zhou, "Hybrid inference network for few-shot SAR automatic target recognition," IEEE Transactions on Geoscience and Remote Sensing, vol. 59, no. 11, pp. 9257-9269, 2021.

[8] G. Chen, L. Wang, and M. M. Kamruzzaman, "Spectral classification of ecological spatial polarization SAR image based on target decomposition algorithm and machine learning," Neural Computing \& Applications, vol. 32, no. 10, pp. 5449-5460, 2020.

[9] M. Touafria and Q. Yang, "SAR-ATR method based on dual convolution capsule network," IET Radar, Sonar \& Navigation, vol. 14, no. 12, pp. 1870-1878, 2020.

[10] Y. Endo, M. Halabisky, L. M. Moskal, and S. Koshimura, "Wetland surface water detection from multipath SAR images using Gaussian process-based temporal interpolation," Remote Sensing, vol. 12, no. 11, 1756 pages, 2020.

[11] A. Maran Beena and A. K. Pani, "Fault detection of complex processes using nonlinear mean function based Gaussian process regression: application to the Tennessee eastman process," Arabian Journal for Science and Engineering, vol. 46, no. 7, pp. 6369-6390, 2020.

[12] M. Khorrami and A. Aghamohammadi, "Thermal conduction in a quasi-stationary one-dimensional lattice system," International Journal of Modern Physics B, vol. 33, no. 17, Article ID 1950178, 2019.

[13] Y.-H. Chen, Y.-Z. Xie, X.-Y. Ge et al., "Vulnerability assessment of equipment excited by disturbances based on support vector machine and Gaussian process regression," IEEE Transactions on Electromagnetic Compatibility, vol. 63, no. 1, pp. 103-110, 2021.

[14] G. B. Chen, Z. Sun, and L. Zhang, "Road identification algorithm for remote sensing images based on wavelet 
transform and recursive operator," IEEE Access, vol. 8, pp. 141824-141837, 2020.

[15] X. Chen, Y. Tian, T. Zhang, and J. Gao, "Differential evolution based manifold Gaussian process machine learning for microwave filter's parameter extraction," IEEE Access, vol. 8, pp. 146450-146462, 2020.

[16] R. Anandaroop and M. David, "Bayesian geophysical inversion with trans-dimensional Gaussian process machine learning," Geophysical Journal International, vol. 217, no. 3, pp. 1706-1726, 2019.

[17] R. Xu, N. Chen, Y. Chen, and Z. Chen, "Downscaling and projection of multi-CMIP5 precipitation using machine learning methods in the upper han river basin," Advances in Meteorology, vol. 217, no. 4, pp. 1706-1726, 2020.

[18] T. Tanti, D. A. Kurniawan, W. Sukarni, E. Erika, and R. Hoyi, "Description of student responses toward the implementation of problem-based learning model in physics learning," JIPF (Jurnal Ilmu Pendidikan Fisika), vol. 6, no. 1, 30 pages, 2021.

[19] M. Klenk and K. Forbus, "Exploiting persistent mappings in cross-domain analogical learning of physical domains," Artificial Intelligence, vol. 195, pp. 398-417, 2013.

[20] X. Ning, K. Gong, W. Li, L. Zhang, X. Bai, and S. Tian, "Feature refinement and filter network for person re-identification," IEEE Transactions on Circuits and Systems for Video Technology, vol. 31, no. 9, pp. 3391-3402, 2021.

[21] X. Ning, W. Li, B. Tang, and H. He, "BULDP: biomimetic uncorrelated locality discriminant projection for feature extraction in face recognition," IEEE Transactions on Image Processing, vol. 27, no. 5, pp. 2575-2586, 2018.

[22] X. Peng, X. Zhang, Y. Li, and B. Liu, "Research on image feature extraction and retrieval algorithms based on convolutional neural network," Journal of Visual Communication and Image Representation, vol. 69, no. 6, Article ID 102705, 2019. 